This is the submitted version of the following article:

Lechuga L., Raptis I., Jorge P., Cusano A.. Preface. Optics and Laser Technology, (2019). 113. : 35 - .

10.1016/j.optlastec. 2018.12.006,

which has been published in final form at https://dx.doi.org/10.1016/j.optlastec.2018.12.006 (c) https://dx.doi.org/10.1016/j.optlastec.2018.12.006. This manuscript version is made available under the CC-BY-NC-ND 4.0 license http://creativecommons.org/licenses/by-nc-nd/4.0/ 


\section{Editors Preface}

Growing demand for biological information in medical diagnostics, healthcare, agriculture, environmental monitoring, and many other application areas resulted in a tremendous interest in the field of biosensors.

Photonic Biosensors, utilizing optical techniques, have thus become one of the most common types of biosensors due their high sensitivity, multi analyte detection potential, compact design, immunity to electromagnetic interference, fast and real-time detection, and remote sensing capabilities. Optical sensing foundations probably dates back hundreds of years when color changes of skin and biological fluids was used in medical diagnostics.

Nowadays, the synergy of nanotechnology with photonics provides a unique combination for the development of next-generation biosensor technologies offering various modalities of biological sensing based on how light interacts with the analytes and specific transduction methods. While fluorescence can get extreme advantages from amplification, providing sensitivity down to single molecules, exploitation of optical interferometry and novel optical surfaces and periodic structures can also yield unprecedented performances without requiring any label.

Exciting and recent technological advances include novel materials and principles in optical detection and transducing mechanisms, lab-on-achip optical platforms and solutions, micro- and nano-optical sensor arrays, methods of surface (bio)functionalization, paving the way for their application in medical diagnostics, pharmacology, health care, intracellular sensing, food analysis, agriculture, environmental monitoring, forensics, defense, and security.

In this context, we have the pleasure to present our special issue on "Photonic Biosensors" through a selection of invited articles authored by leading authorities in their respective fields providing a broad and exciting picture of the main innovations pertaining to photonic biosensors and devices, which are related to photonic transduction principles, engineering, and technology highlighting the impact and relevance to face critical problems and open issues in the fields of medicine, biology, and biotechnology.

With a view towards performance enhancement, Wangüemert-Pérez et al. present an extensive analysis, both from a practical and a theoretical perspective, of current integrated photonic biosensors, and analyze how subwavelength structures can be exploited to enhance their sensitivity. This study is not restricted just to the near-infrared band as also determines the sensing capabilities of the suspended silicon waveguides with subwavelength metamaterial cladding working in the mid-infrared range. These waveguides have been recently proposed to cover the full transparency window of silicon, where the fingerprint spectral region of many molecules takes place and so a plethora of evanescent field absorption-based applications will be developed in the near future.

Optical fibers due to their extreme flexibility and versatility provide the technological basis for the development of novel and improved low-cost surface plasmon resonance (SPR) platforms for specific applications. In this context, Zeni and co-workers demonstrated an intriguing optical fiber assisted plasmonic platform coupled with functionalised molecularly imprinted polymers as biochemical sensing device. The innovative platform combines the advantages of slab waveguides and optical fibers providing distinctive features in controlling the number of interacting light modes and thus the final performances of the device.

Biomimetic and bio-inspired technologies arising from a flow of ideas from the biological sciences into disciplines as engineering, optics and photonics, chemistry, materials science, physics have recently spurred an increase in efforts aimed at emulating nature's most impressive design strategies in man-made technology. Here, Martín-Palma et al. review the field of Biomimetic photonic structures for optical sensing showing how "Learning from nature how to build devices with a specific functionality" has the distinct advantage of capitalizing on thoroughly scrutinized, performance-optimized outcomes of millions of years of evolution and natural selection in living systems.

Labeling of biological matter is a key technique enabling visualization and real-time tracking of dynamic processes beyond the diffraction limit. While the precision of fluorescence-based localization microscopy is limited by signal saturation, a new generation of tiny scattering labels (such as gold nanoparticles) have brought the localization precision below the size of a single protein. However, the size of scattering labels is also the most obvious handicap, as they are usually significantly larger than the resulting localization precision. With a view towards unprecedented performances, Pilarik and associates here address this inconsistency and demonstrate detection and precise localization of gold nanoparticles of only $\mathbf{2} \mathrm{nm}$ in diameter using interferometric detection of scattering, foreseeing new methodologies and concepts to study biomolecular systems in the future.

Recently, nanoaperture optical tweezers have extended the range of optical tweezers to dielectric particles below $50 \mathrm{~nm}$ in size allowing the efficient trapping of proteins, DNA fragments and other biomolecules, as well as small viruses. Here, Gordon et al. review this intriguing field with particular emphasis on the potential of these technological platform to obtain direct measurements about the structure and dynamics of biomolecules, which is expected to be immediately useful in verifying the biophysical processes involved in new drug candidates. Significant advances have been made in nanofabrication, and it is now envisioned that "nanopipettes" may soon be available where multiple nanoapertures are integrated on fibers to enable parallel well-plate interrogation in an automated fashion. The review also highlights the main challenges that still remain in the wide scale adoption of this technique.

New biological sensors are expected to be addressed by photonic integrated technologies, targeting the monitoring of vital parameters, from skin temperature and sweat analysis to continuous monitoring of pulse, respiratory and cardiovascular auscultation, or breath gas analysis. In this context, Porcel and associates propose a fascinating review paper describing the silicon nitride material platform for building photonic waveguides and circuits, with a clear focus on visible operation wavelengths and biophotonic applications. A compilation of state-of-the-art results on SiN photonic devices is here reported, evaluating key performance indicators of several examples. 
Ultra-low detection limits for small molecules quantization has been recently demonstrated by Nabok et al. by using the combination of two optical methods, e.g. total internal reflection ellipsometry and LSPR transducers based on nano-structured gold films and planar waveguides operating as polarization interferometers. This judicious combination allows the detection of low molecular weight analytes, such as mycotoxins, in concentrations down to $0.01 \mathrm{ng} / \mathrm{mL}$ (10 ppt) using a simple direct immunoassay format. The limitations of LSPR due to a short evanescent field decay length can be overcome using small bio-receptors, such as half-antibodies and aptamers. The achieved sensitivity of detection of mycotoxins $(0.01 \mathrm{ppb})$ is sufficient for the analysis with this method of agriculture products, food and feed on the presence of mycotoxins.

With a view towards applications in clinically relevant scenarios, Mendoza and coworkers here illustrate the main building blocks of an electrophoretic plasmonic nanopore (EPIGeneS) biochip sequencing platform. This study sets the foundations to further investigate the methodologies needed to optimize the electrophoretically control and slow the translocation rate of DNA through the torturous nanopore structures of the chip. This is done in order to enable the measurement of DNA molecule single nucleobases as it translocates from the nanofluidic conduit channels through the nanoporous "roof" structure of the chip to enable the spectral visualization of the DNA base pair signatures using surface enhanced Raman imaging techniques. This study opens new avenues for the development of high throughput convenient and portable EPIGenes biochip device that will enable the rapid, reliable, and automated DNA sequencing of an entire human genome at a cost suitable for large scale usage in biological research and medicine.

Multiple limitations still remain to current leukemia diagnostic protocols for the morphological analysis of cells in a blood sample, including low sensitivity, photobleaching and limited multiplexing for the fluorescence based selective detection of cell phenotypes. In this context, De Luca and co-workers review the efforts towards developing strategies based on Raman Spectra and SERS for label-free biochemical identification, classification and imaging of cells from the peripheral blood as a potential diagnostic tool for leukemia. Intrinsic Raman markers associated with DNA, proteins and lipids are demonstrated to allow the specific cell identification and classification, based on their maturation/differentiation level, with high accuracy (about $97 \%$ ). The acquisition of single-point Raman spectra from cell volumes of few $\mathrm{fL}$ can take less than a second, and could be used for automated label-free analysis of a large number of cells from blood samples.

All the above described contributions are a clear and evident glimpse of the wealth of recent publications and hot topics in the rapidly growing field of Optical Biosensors. The synergy of nanotechnology with photonics, or nanophotonics, is opening new avenues for the development of the next-generation of photonic biosensor technologies that will impact life sciences research and healthcare. 